\title{
Bowel endometriosis: case report
}

\author{
Ajith Lamahewage ${ }^{1}$, JPM Kumarasinghe ${ }^{2}$, NG Ranawaka ${ }^{3}$ \\ ${ }^{1}$ Senior Lecturer, Department of Pathology, ${ }^{2}$ Senior Lecturer, Department of Surgery, ${ }^{3}$ Lecturer, \\ Department of Pathology, Faculty of Medicine, University of Ruhuna, Galle.
}

\section{Introduction}

Endometriosis is defined as the presence of endometrial glands and/or stroma in extra uterine sites. It is principally a disease of women in active reproductive life. This disorder often causes infertility, dysmenorrhoea, pelvic pain, and symptoms related to the affected organs. It occurs in the following sites in descending order of frequency: ovaries, uterine ligaments, rectovaginal septum, pelvic peritoneum, laparotomy scars and rarely in umbilicus, vagina, bladder and bowel. Three potential explanations of the origin of endometriosis are; the regurgitation-implantation theory, the metaplastic theory and the vascular or lymphatic dissemination theory. In addition hormonal and immune genetic factors may play a role for the susceptibility to develop endometriosis [1].

\section{Case Report}

A 37 year-old female was referred to the surgical casualty ward with a vague pain in the right side of the abdomen. The clinical diagnosis was an acute appendicitis. Conventional appendectomy was attempted but a mass lesion involving the wall of the ileo-caecal junction was found. Therefore, an exploratory laparatomy was carried out.

Part of the ascending colon, caecum along with the appendix and a part of ileum was removed and end to end anastamosis was made.

Pathological specimen consisted of a segment of the ascending colon, caecum along with the appendix and a segment of ileum. A white, constricting lesion was seen involving the wall of the bowel at the ileocael junction which appeared to involve the serosa, too. The lesion measured $4 \times 2 \times 2 \mathrm{~cm}$. Appendix looked macroscopically normal. Eleven enlarged mesenteric lymph nodes were recovered.

Histological sections from the constricting lesion in the ileo-cecal junction showed several foci of endometrial glandular structures accompanied by endometrial stroma embedded in muscularis propria (Figure 1). They were lined by a single layer of columnar epithelium with basally located nuclei. No atypical features were noted. 
Occasional mitoses were seen in the stroma. Intestinal mucosa of the ileum and caecum showed a moderate chronic inflammatory cell infiltration. Ascending colon and appendix were microscopically normal. All the eleven lymph nodes showed features of reactive hyperplasia.

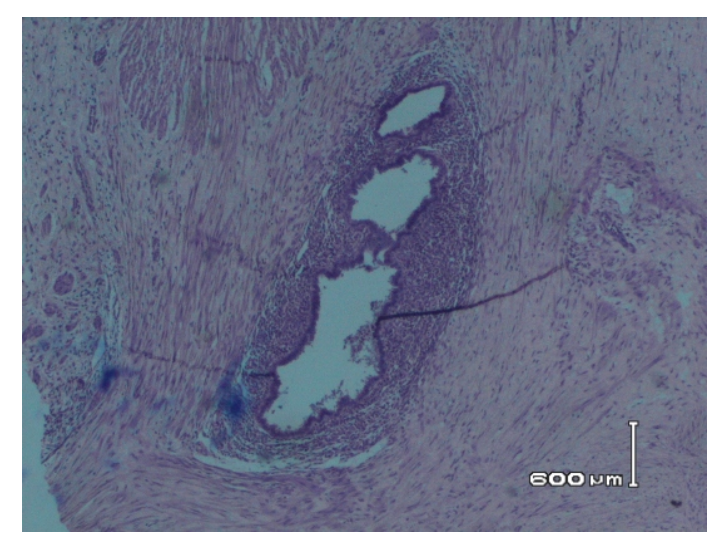

Figure 1 - Histological section of the lesion in the ileo - caecal junction

\section{Discussion}

Bowel endometriosis is very rare. It is usually an incidental finding in the gut, but some examples present themselves as an obstructing tumefactive mass that closely simulates an intestinal neoplasm [2]. Although some women with bowel endometriosis may be asymptomatic, the majority of them develop a variety of gastrointestinal complaints. Except for rectal nodules bowel endometriosis cannot be diagnosed by physical examination. Therefore, imaging techniques such as double contrast barium enema, transvaginal ultrasonography; rectal endoscopic ultrasonography, magnetic resonance imaging (MRI) and multi slice computed tomography enterolysis should be used [3].

Medical management of bowel endometriosis is currently speculative. Several studies demonstrated an improvement in quality of life after surgical excision of the lesion.

Bowel endometriotic nodules can be removed by various techniques; mucosal skinning, nodulectomy, full thickness disc-resection and segmental resection of the bowel [2].

\section{References}

1. HC Anderson, Endometriosis. In: W.St. Symmer's. Systemic Pathology, $3^{\text {rd }}$ Edition, Vol. 6, Churchill Livingstone, 1990; 241-62.

2. Patrick L, Fitzgibbons. Pseudoneoplastic lesion of alimentary treat. In: Mark R Wick, Peter A Humphrey, Jon H Ritter. Pathology of Pseudoneoplastic Lesions, $1^{\text {st }}$ Edition, Lippincott, Raven: 1997: 149-150.

3. Juan Rosai. Female genital trent. In: Juan Rosai. Ackerman's Surgical Pathology, $9^{\text {th }}$ Edition, Vol 2: Mosby, 2004; 1569-635. 